\title{
A Study on the Best Conventional Shapes for Composite Repair Patches
}

\author{
Leonel Echer * (D), Carlos Eduardo de Souza ${ }^{b}$ (D), Rogério José Marczak ${ }^{a}$ \\ ${ }^{a}$ Universidade Federal do Rio Grande do Sul, Departamento de Engenharia Mecânica, \\ Rua Sarmento Leite, 90050-170, Porto Alegre, RS, Brasil. \\ ${ }^{b}$ Universidade Federal de Santa Maria, Departamento de Engenharia Mecânica, \\ Av. Roraima, 97105-900, Santa Maria, RS, Brasil.
}

Received: June 22, 2021; Revised: August 16, 2021; Accepted: August 25, 2021

\begin{abstract}
Adhesively bonded repair patches are an excellent approach for repairing locally damaged composite components. If correctly applied, fiber-reinforced patches may restore/increment the mechanical response of damaged laminates without significantly increasing the structure's mass or altering its geometry. However, in order to take full advantage of this repairing technique, one must employ patches with a minimal surface area and maximum efficiency in incrementing the strength of the component. The present work aims to study optimum-based patch shapes for conventional repair geometries, namely rectangular and elliptical. Shell Finite Elements models were used to simulate a parent plate, which is a rectangular flat laminate with a central trespassing damage region. Unbalanced single-ply patches were modeled on the upper surface of the damaged laminate. The patches' efficiency was computed as its capability in restoring the modal response of the repaired component to its undamaged configuration. Sequential linear programming was employed alongside shell finite element models to obtain optimal geometrical parameters for the patches' shape. The study cases comported two different boundary conditions and two stacking sequences. The optimum-base repair patches were defined regarding size and fiber orientation angle.
\end{abstract}

Keywords: Repair patch, damaged laminate, structural optimization, modal response, shell FE.

\section{Introduction}

In the past decades, the aerospace industry remarkably increased the use of advanced materials in both primary and secondary structures. Moreover, modern aircraft components are usually broad and highly integrated. When damaged, the complete replacement of this sort of components may be economically impracticable or even unfeasible. This scenario unleashed an unprecedented demand for efficient Maintenance Repair and Overhaul, MRO, techniques aiming for the repair of composites ${ }^{1}$.

In the context of structural repair, adhesively bonded fiber-reinforced patches appear as an excellent approach for locally restoring the mechanical response of damaged composite structures. The appeal for these patches is due to their capability to restore/increment the mechanical response of damaged components without significantly increasing the structure's inertia or altering its aerodynamics. However, the use of structural repair patches is still very limited in the aerospace industry. This restriction may be credited to both aviation rules and certification, and technology development ${ }^{2}$.

\subsection{Fiber-reinforced repair patches}

The first applications of fiber-reinforced repair patches date from the late ' $60 \mathrm{~s}^{3-5}$. However, these early studies were mainly empirical and limited to military applications; focusing only on reducing the Stress Intensity Factor (SIF)

*e-mail: leonel.echer@ufrgs.br and preventing the crack growth of damaged metallic sheets ${ }^{4}$. It was only during the early 90's that the use of adhesively bonded repair patches was extended to civil aviation ${ }^{6}$.

After the late '90s, the study of the patch's geometrical shape gained a prominent role. Different authors already demonstrated that smaller patch surfaces reduce drawbacks such as humidity absorption and residual thermal stress due to the curing process. A small patch also implies slighter changes in the component's inertia and aerodynamics, which is highly desirable ${ }^{7,8}$. However, defining a repair patch, with a minimal surface area, without affecting its capability to restore the mechanical response of a damaged component is not trivial. This statement is particularly true for composites materials, which present heterogeneous material properties and complex failure modes. Moreover, the conventional patch shapes successfully employed for metallic components may not be suitable for composites.

\subsection{Unconventional shapes for repair patches}

Over the years, different unconventional shapes, i.e., the ones that are not geometric plane shapes, have been proposed aiming to improve the capability of smaller patches. Some of these geometrical shapes are presented in Table 1. Despite the differences between these patch shapes, all share the same basic idea of priming for material distribution along with the surroundings of crack tips, which is somewhat intuitive in the repairing of metals. This concept was proposed by ${ }^{9}$ and 
Table 1. Different repair patch strategies.

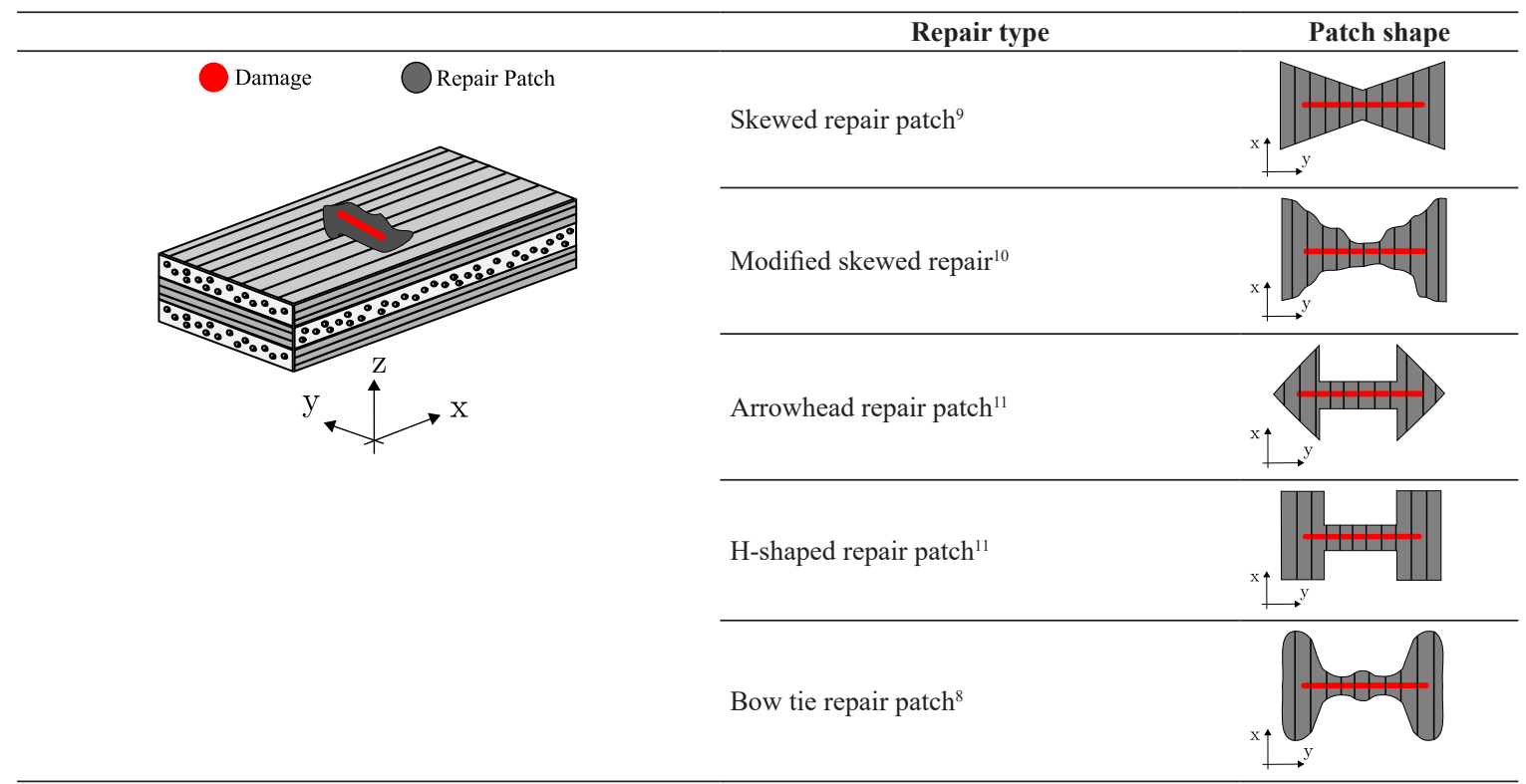

later extended to other shapes using experimentation and optimization with genetic algorithms.

The shapes presented in Table 1 might considerably reduce the patch area. However, reproducing these unconventional shapes in a real-life application may be impractical. Moreover, all the shapes of Table 1 assume the patch fibers orientation

as perpendicular to the crack/damage normal axis. This premise is valid for most crack-like damage in metallic components with isotropic behavior. However, it may not be accurate to composite materials ${ }^{12,13}$. Moreover, the patch fibers orientation $\phi$ plays a significant role in the capability of composites repair in supporting loading.

\section{Repair Patch Shape Optimization}

The design of an optimum repair patch for composites must consider: (a) minimal surface area $\Omega_{r}$ necessary for restoring the mechanical response of the damaged component, and (b) fibers orientation angle $\phi$ that allows maximum efficiency in transferring strength to the repaired structure. The objective of the present work lies in proposing optimum-based patch shapes incorporating these two distinct conditions.

To achieve these objectives, finite element models were iteratively solved within an optimization algorithm as part of a two-phase optimization formulation. Shell finite element models were employed in the simulation of the damaged laminate panels with adhesively bonded repair patches. An interior-point algorithm was used to solve the linearized problem and obtaining optimum parameters for $\Omega_{r}$ and $\phi$.

\subsection{Problem of study}

The study model was defined aiming to simulate a composite panel. It was considered as a flat rectangular laminate with dimensions $l \times l / 2$, where $l=1 \mathrm{~m}$. The component was modeled as an eight-layer laminate with ply thickness $t_{i}=0.4 \mathrm{~mm}$.
A centered damage region was considered as rectangular with dimensions $d \times d / 4$, where $d=l / 20$. Table 2 presents the geometrical configuration of the damaged laminate and its material mechanical properties.

The damage was considered trespassing and was modeled as a severe local degradation of the parent plate mechanical properties. This level of material deterioration was defined as four orders of magnitude, $\mathrm{E}_{i i} \times 10^{-4}$ and $\mathrm{G}_{i j} \times 10^{-4}$, which is a value high enough to avoid numerical problems, $\mathrm{E}_{i i}, \mathrm{G}_{i j} \rightarrow 0$, and low enough to model severe loss of mechanical strength. For all the numerical models the damage region differs from the rest of the geometry only regarding elastic and shear modulus, $\mathrm{E}_{i i}$ and $\mathrm{G}_{i j}$. By doing this, the overall mass of the laminate is not altered, i.e., the damage affects only the structure's strength.

Two different stacking sequences were considered, namely [0/45/-45/90]s and [45/-45/0/90/60/-30/90/-45]. The symmetric stacking sequence was selected to simulate a conventional commercial composite. The unsymmetrical one was tested to evaluate the influence of the stacking sequence over the optimized parameters.

Two boundary conditions were simulated, fully clamped (FC) and simply supported (SS), as presented in Table 2 . These choices were made aiming to simulate the parent plate as a representation of a composite panel in an aircraft fuselage. For such a scenario, a more realistic boundary condition would be the use of springs acting as stiffeners on all the perimeter of the parent plate. However, for simplification purposes, only the fully clamped and simply supported conditions were tested.

The repair patch was considered as a single-ply with the thickness $t_{r}=t_{i}$, and it was modeled as an unbalanced unidirectional patch acting on the upper surface of the damaged plate. Two traditional patch geometries were selected to be optimized, namely rectangular and elliptical. 
Table 2. Laminate mechanical properties.

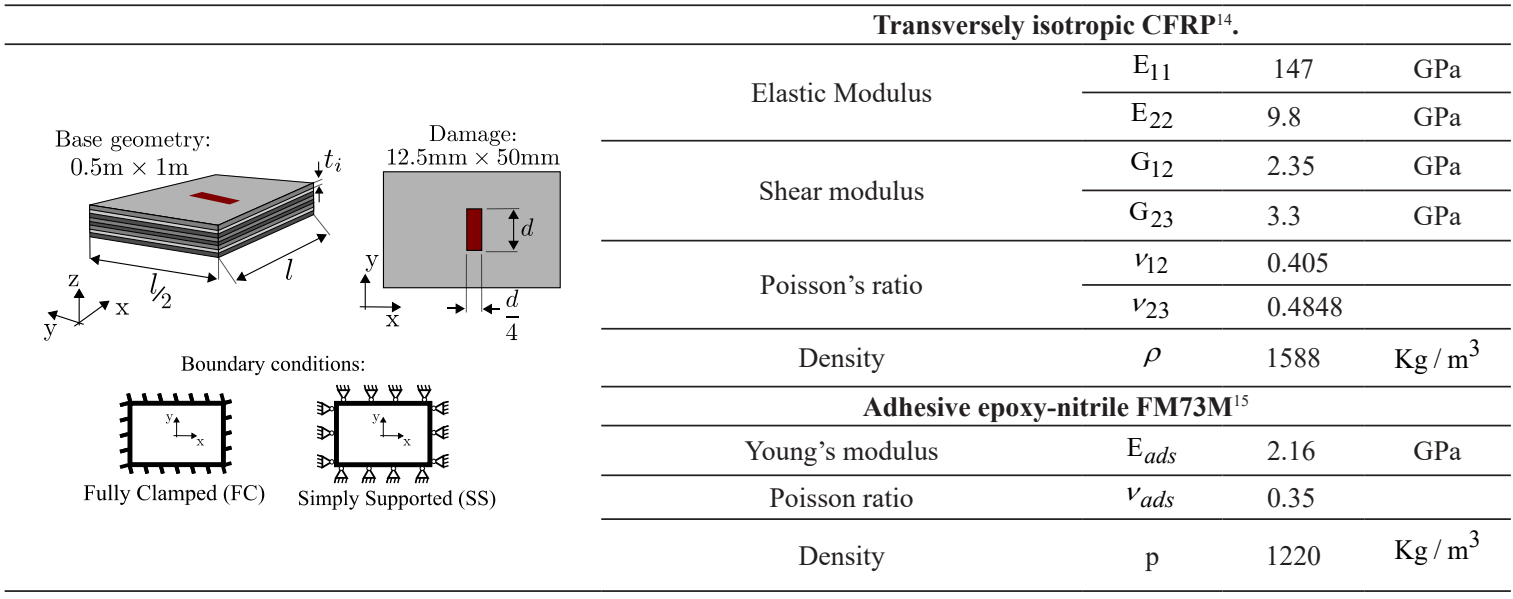

Table 3. Base shapes tested.

$\begin{gathered}\text { Elliptical Patch design } \\ \text { variables, } x_{i}:\end{gathered}$
Repair Shapes
- Fibers orientation $\phi$

Table 3 presents these geometrical shapes and their respective design variables.

\subsection{Optimization problem formulation}

In order to optimize the patch shapes presented in Table 3, it was adopted a formulation with two optimization phases. In the first phase, the optimum $\phi$ was evaluated for each base repair shape geometry. The second phase employed this result as entry data and evaluated the optimal shape parameters, design variables $x_{i}$.

The objective of the first optimization phase was to obtain the value of $\phi$ that maximizes the repair strength. A direct approach considering the strength or the stiffness of the repaired component and its sensitivity to the design variables would be extremely complex. However, an optimization formulation for the structure's mechanical behavior regarding its modal response is far less intricate. By using this approach, an objective function $O b j_{\phi}$ was defined in such a way that its minimization implied maximizing the first natural frequencies of the repaired structure. Moreover, it is reasonable to assume a straight relationship between the adopted approach and the structure's stiffness response. This statement is particularly true considering that: (i) the mass of the repair patch is not capable of significantly altering the repaired structure inertia and (ii) the damage does not affect the components mass. This problem was formulated as a single design variable optimization and stated as $\underset{\phi}{\min \text { imize }_{\phi} O b j_{\phi}}=\sum_{i=1}^{3} \frac{f_{i}}{\overline{f_{i}}}$, subjected to $-\pi / 2 \leq \phi \leq \pi / 2$,

where, $\overline{f_{i}}$ is the $i$-th natural frequency of the repaired component and $f_{i}$ is the $i$-th natural frequency of the undamaged structure, comparison solution.

After solving the optimization problem of Equation 1, the optimized value of $\phi$ was employed as entry data for the second optimization. This second phase aimed to obtain the minimal repair patch area, $\Omega_{r}$, capable of restoring the repaired component modal response to its undamaged state. The objective function of this second optimization was defined as the error for the first natural frequencies of the comparison solution,

$\operatorname{Obj}_{f}=\sqrt{\sum_{i=1}^{3}\left(\frac{f_{i}-\overline{f_{i}}}{f_{i}}\right)^{2}}$,

resulting in the following optimization formulation

$\underset{f}{\operatorname{minimize}} \mathrm{Obj}_{f}$, subjected to $x_{l} \leq x_{i} \leq x_{u} \quad \forall x_{i} \in \Omega_{r}$,

where the side constraints $x_{l}$ and $x_{u}$ are the lower and upper limits for $x_{i}$, respectively. Also, the feasible domain of solution, $\Omega_{r}$, was defined as the edges of the parent plate. This implied in giving the freedom to the repair patches to assume shapes as wide as the parent plate. 
The two-phase optimization process was carried out employing Linear Programming, LP, to iteratively solve a shell Finite Element (FE) model. Commercial software were used for implementing all the mathematical programming ${ }^{16}$, and also in the solution of the FE models ${ }^{17}$. LP was selected due to its simplicity, especially for side-constrained problems. The modal analysis employed a Block Lanczos method for eigenmodes extraction. The FE solver was set for the evaluation of the first nine modes, and only the first three were used. The sensitivity analysis was carried out through a numerical approximation using Forward Finite Differences with a disturbance of $0.1 \%{ }^{18}$, and the optimization problem was linearized employing a Taylor Series expansion. An interior-point algorithm was used to solve the resulting problem ${ }^{19}$. A step-length, $\alpha_{i}$, modifier based on past iterations, $i-1$, and $i-2$, was employed for the design variables updating ${ }^{20}$.

\section{Results}

Table 4 summarizes the optimal values of $\phi$ for each case of study. The number of iterations, $N$, until convergence, and the initial random guess for each optimization problem are presented as well.

The parent plate with symmetric stacking sequences presented similar results, a difference of $\approx 3^{\circ}$, independently of the boundary condition. However, the case with an unsymmetrical stacking sequence presented an optimum $\phi$ for the Simply Supported condition that was more than 2.3 times greater than the one obtained for the Fully Clamped condition.

Due to its quasi-isotropic behavior, associated with the aspect ratio of the damaged region, the optimal solutions for the component with symmetrical stacking sequence presented $\phi$ almost perpendicular to the damage central axis. However, for the component with odd stacking sequences, this phenomenon is not visible, despite the damage shape. Furthermore, for the repair of composites, the definition of the most effective fibers orientation $\phi$ is not as trivial as it may be for cracked metallic components. This statement is particularly true for laminates with unsymmetrical stacking sequences or with odd damage shapes.

Table 5 presents the eigenvalues for the four analyzed cases. These values, alongside the optimized values of $\phi$, were the comparison solutions employed within Equation 3 to solve the second stage of the parametric optimization problem.

\subsection{Stacking sequence [0/45/-45/90]s - fully clamped boundary conditions}

The optimum-based repair shapes for the fully clamped laminate with symmetrical stacking sequence are presented in Figures 1 and 2. The results are presented in terms of optimization evolution for the design variables and convergence.

Table 4. Optimum $\phi$.

\begin{tabular}{|c|c|c|c|c|}
\hline & \multicolumn{2}{|c|}{ Laminate $[0 / 45 /-45 / 90] s$} & \multicolumn{2}{|c|}{ Laminate $[45 /-45 / 0 / 90 / 60 /-30 / 90 /-45]$} \\
\hline & Fully Clamped & Simple Supported & Fully Clamped & Simple Supported \\
\hline Iterations & 25 & 25 & 18 & 27 \\
\hline Initial Guess, $\phi_{1}$ & $-33.23^{\circ}$ & $32.80^{\circ}$ & $36.43^{\circ}$ & $80.30^{\circ}$ \\
\hline$\phi_{\text {otim }}$ & $-3.15^{\circ}$ & $-6.02^{\circ}$ & $32.16^{\circ}$ & $13.69^{\circ}$ \\
\hline
\end{tabular}

Table 5. Modal response $f_{\boldsymbol{i}}$ for the undamaged composite panels.

\begin{tabular}{cccccc}
\hline & \multicolumn{2}{c}{ Laminate $[\mathbf{0} / \mathbf{4 5} / \mathbf{- 4 5 / 9 0 ]}$} & & \multicolumn{2}{c}{ Laminate $[\mathbf{4 5 / - 4 5 / 0 / 9 0 / 6 0 / - 3 0 / 9 0 / - 4 5 ] ~}$} \\
\cline { 2 - 3 } & Fully Clamped & Simple Supported & & Fully Clamped & Simple Supported \\
\hline$f_{1}$ & $65.46 \mathrm{~Hz}$ & $54.19 \mathrm{~Hz}$ & & $83.59 \mathrm{~Hz}$ & $62.41 \mathrm{~Hz}$ \\
\hline$f_{2}$ & $104.75 \mathrm{~Hz}$ & $85.62 \mathrm{~Hz}$ & & $108.67 \mathrm{~Hz}$ & $87.15 \mathrm{~Hz}$ \\
\hline$f_{3}$ & $157.59 \mathrm{~Hz}$ & $115.29 \mathrm{~Hz}$ & & $148.64 \mathrm{~Hz}$ & $125.73 \mathrm{~Hz}$ \\
\hline
\end{tabular}

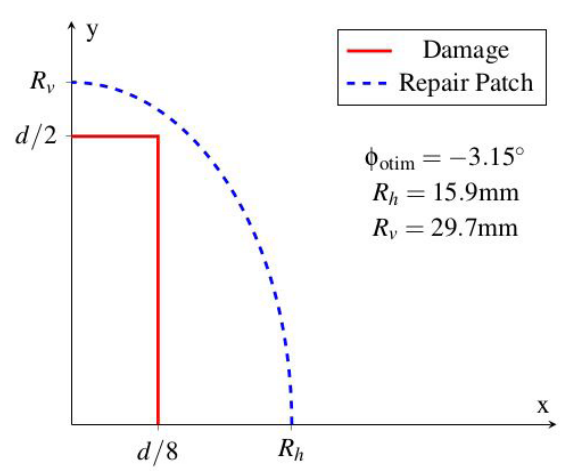

(a) Repair patch and damage region - 1/4 symmetry.

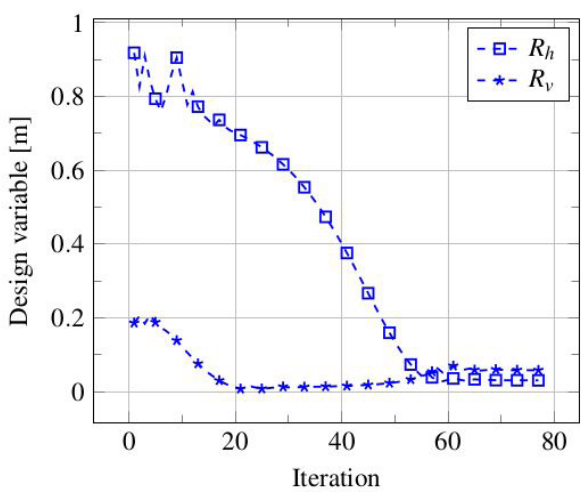

(b) Convergence and optimization evolution.

Figure 1. Elliptical repair patch - FC with symmetric stacking sequence. 
The optimized elliptical patch, Figure 1a, fully covered the damaged region with a total area of $1,482.9 \mathrm{~mm}^{2}$. Analogously, the rectangular patch presented a similar optimized configuration with a total area of $1,624.0 \mathrm{~mm}^{2}$ . Both repair patches extended through the longitudinal direction, $\mathrm{x}$-axis, implying that the modal amplitude of the damaged component was amplified in this direction as well. This effect was credited to two factors: (i) restraining of the rotational degrees of freedom by the boundary condition; and (ii) aspect ratio of the parent plate.

\subsection{Stacking sequence [0/45/-45/90]s - simply supported boundary conditions}

Figures 3 and 4 present the optimum-based repair shapes for the laminate with symmetrical stacking sequence and simply supported boundary conditions. Once again, the results are presented in terms of optimization evolution for the design variables and convergence.

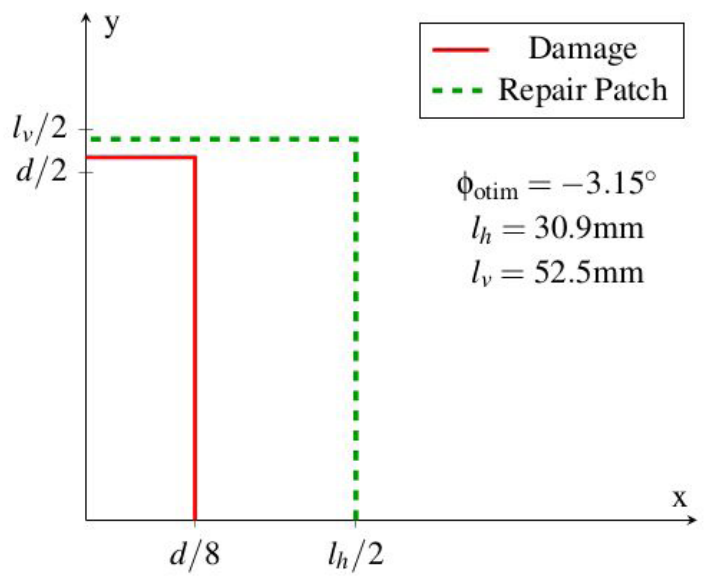

(a) Repair patch and damage region - 1/4 symmetry.
The damage presence results in the amplification of the modal displacement. The repair patches act overlapping the most affected region and restoring the structure's modal response. The simply supported boundary conditions allowed this amplification to be very concentrated in the surroundings of the damaged area, which resulted in smaller repair patches slightly covering the damaged area. This fact is particularly visible for the optimized elliptical patch, Figure 3a, which has a total area of $1,093.3 \mathrm{~mm}^{2}$ and intersects the perimeter of the damaged zone. The optimized rectangular patch is slightly bigger with $1,238.8 \mathrm{~mm}^{2}$.

\subsection{Stacking sequence [45/-45/0/90/60/-30/90/-45] - fully clamped boundary conditions}

The optimum-based repair shapes for the fully clamped laminate with an unsymmetrical stacking sequence are presented in Figures 5 and 6. Once more, the results are

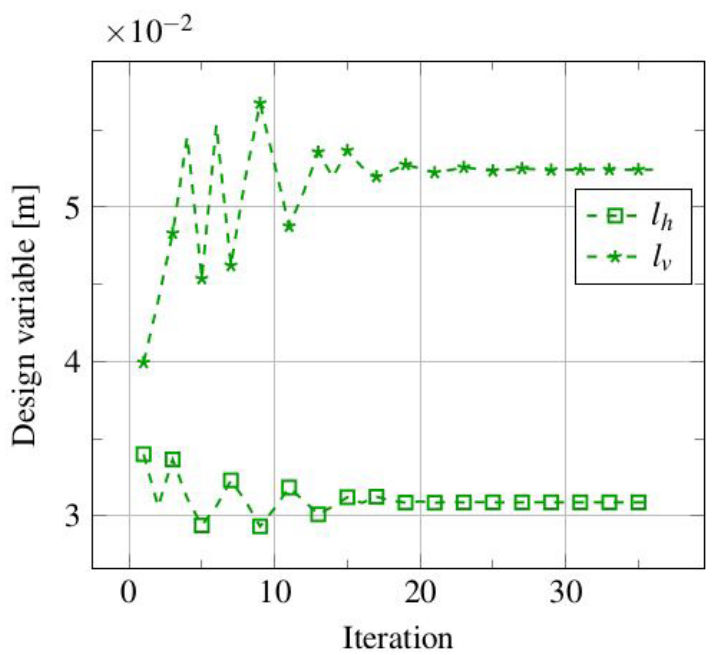

(b) Convergence and optimization evolution.

Figure 2. Rectangular repair patch - FC with symmetric stacking sequence.

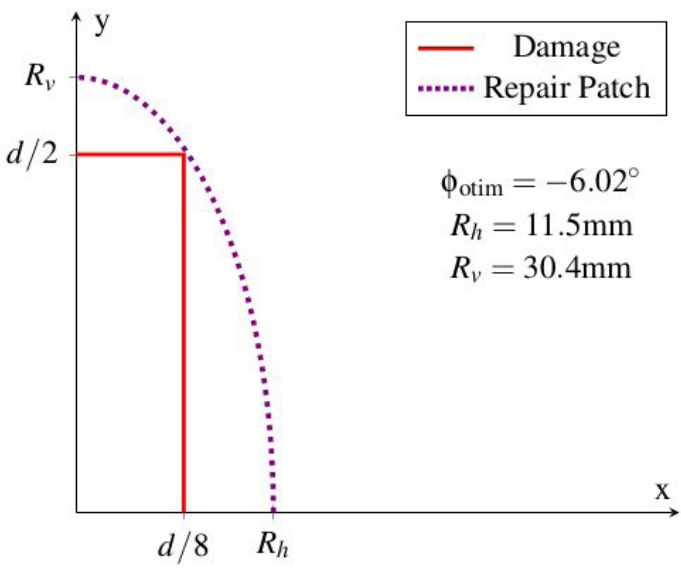

(a) Repair patch and damage region - 1/4 symmetry.

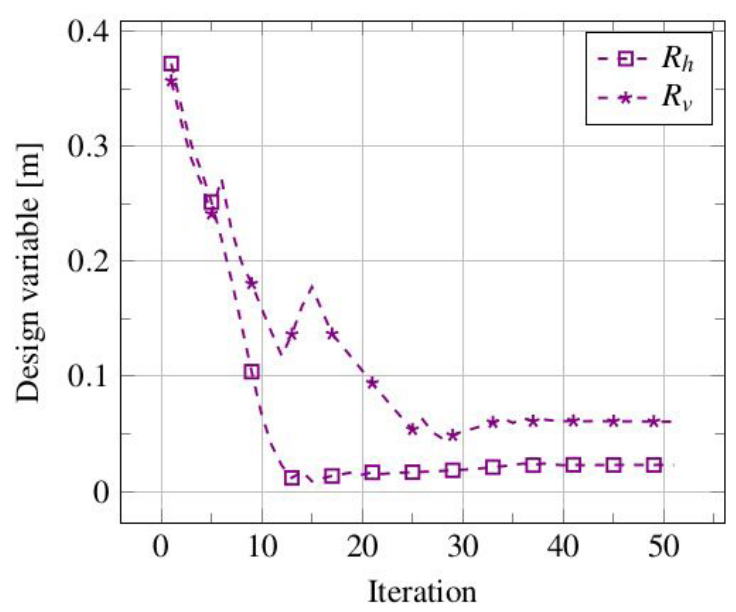

(b) Convergence and optimization evolution.

Figure 3. Elliptical repair patch - SS with symmetric stacking sequence. 


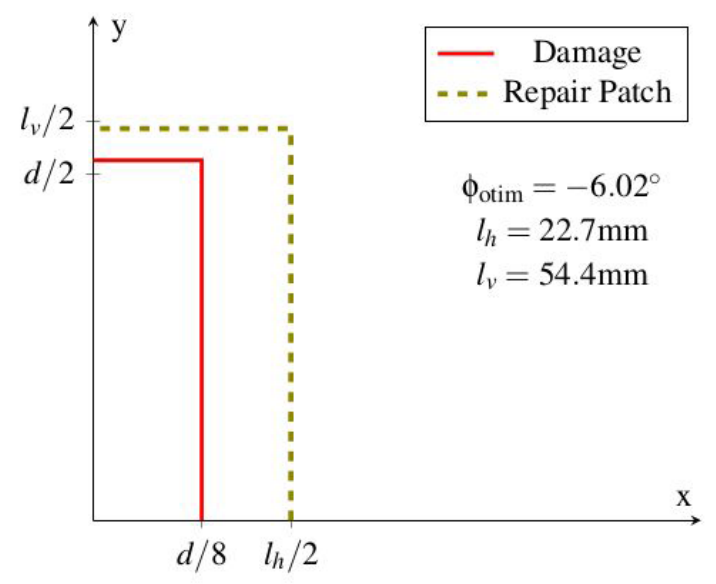

(a) Repair patch and damage region - $1 / 4$ symmetry.

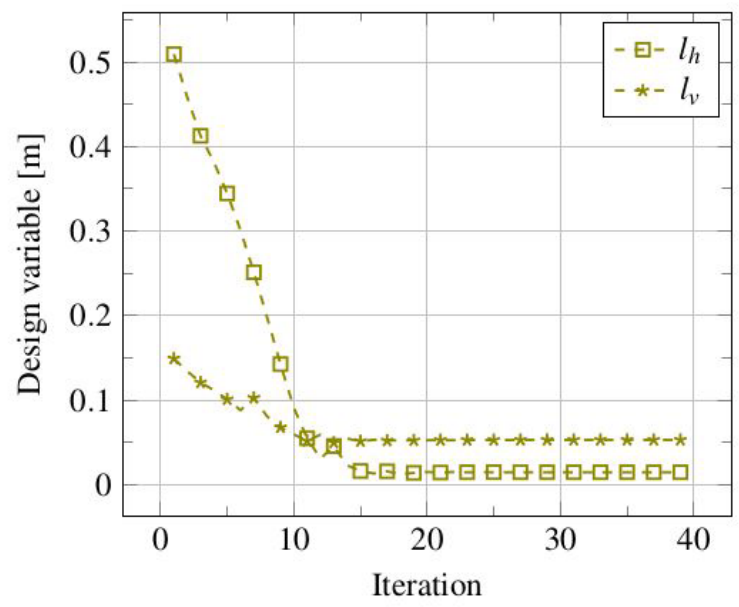

(b) Convergence and optimization evolution.

Figure 4. Rectangular repair patch - SS with symmetric stacking sequence.

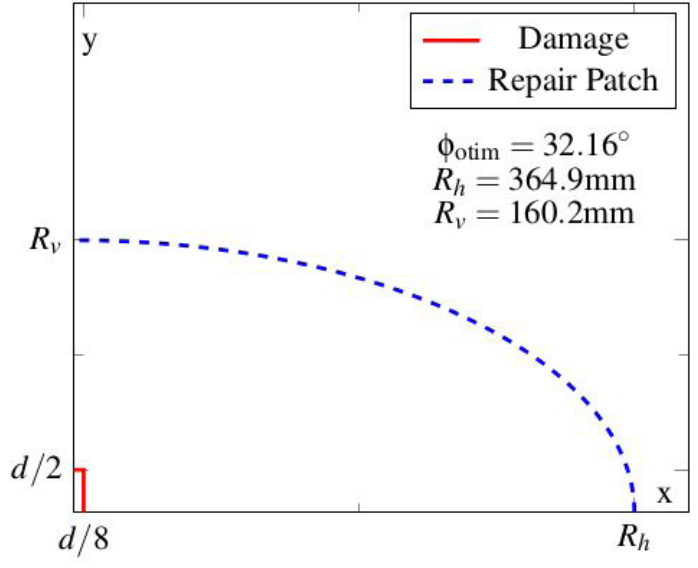

(a) Repair patch and damage region - 1/4 symmetry.

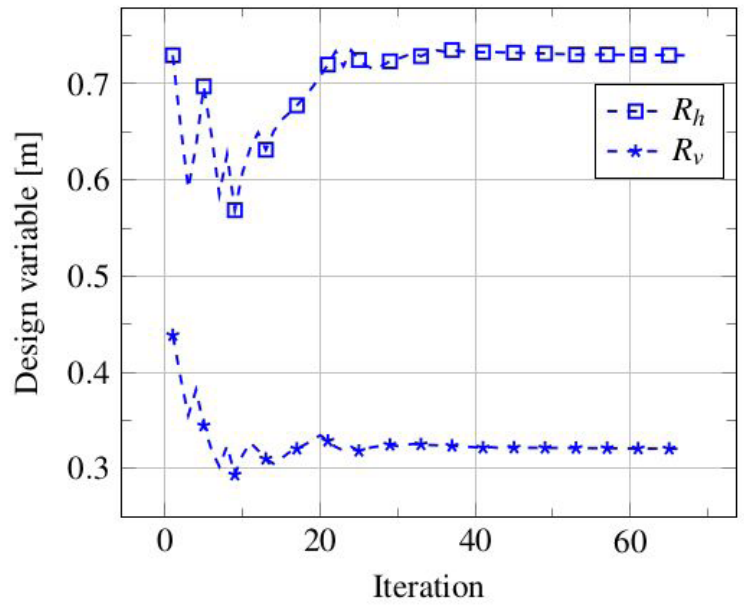

(b) Convergence and optimization evolution.

Figure 5. Elliptical repair patch - FC with an unsymmetrical stacking sequence.

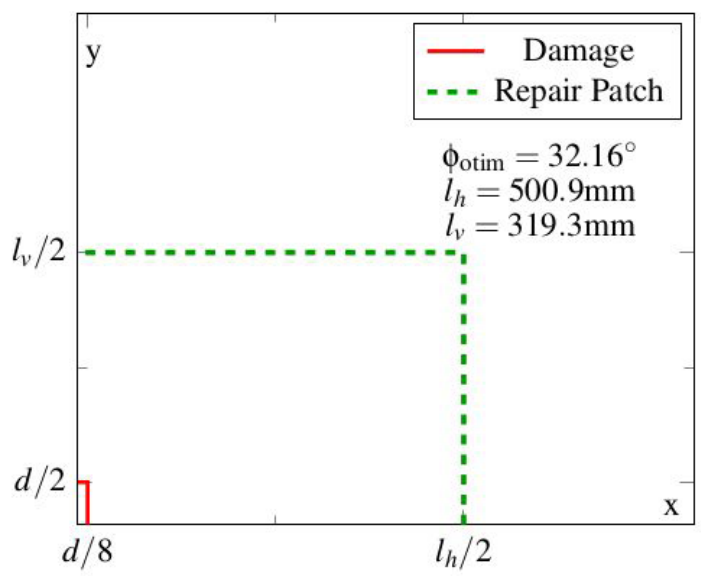

(a) Repair patch and damage region - 1/4 symmetry.

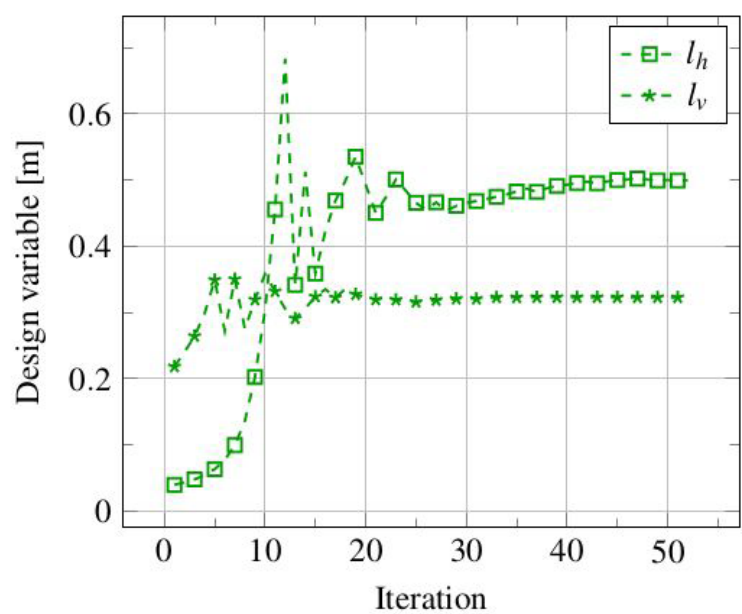

(b) Convergence and optimization evolution.

Figure 6. Rectangular repair patch - FC with an unsymmetrical stacking sequence. 
presented in terms of optimization evolution for the design variables and objective function.

The quasi-isotropic effect is not presented by this stacking sequence, which implies the spreading of the modal displacement amplification along a broad region of the parent plate. Unlike the cases for the symmetric stacking sequence, the optimized patches for the unsymmetrical laminate need to cover a larger area to suppress this amplification caused the damage presence. Moreover, due to the fully clamped boundary conditions, the patches extend in both directions of the Cartesian plane.

The optimized elliptical patch, Figure 5a, presented an area of $18,366.8 \mathrm{~mm}^{2}$, and considerably extended its length in the $\mathrm{x}$-axis direction. The optimized rectangular patch was smaller with $15,994.6 \mathrm{~mm}^{2}$ and an aspect ratio similar to a square. This is attributed due to the modal displacement amplification along the diagonal of the parent plate. This effect, induced by the unsymmetrical stacking sequence, favored the rectangular patch.

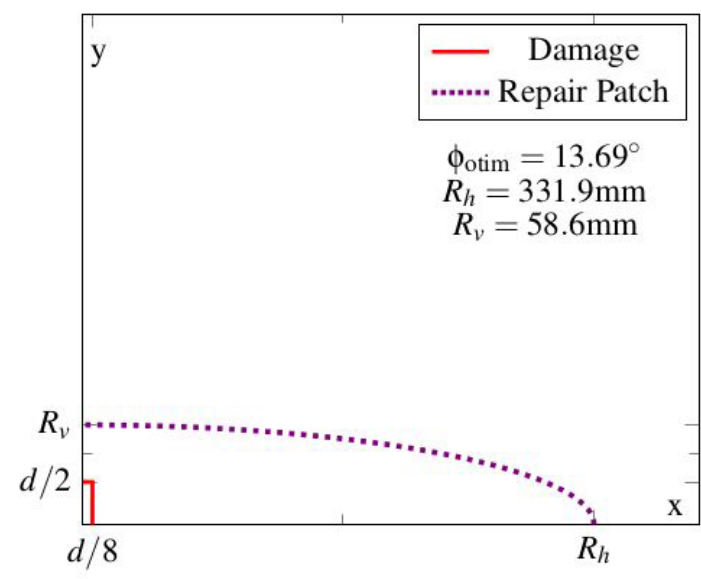

(a) Repair patch and damage region - 1/4 symmetry.

\subsection{Stacking sequence [45/-45/0/90/60/-30/0/-45]- simply supported boundary conditions}

The optimized patch shapes for the simply supported unsymmetrical laminate are presented in Figures 7 and 8. The optimization evolution for both elliptical and rectangular repairs is presented as well.

The simply supported boundary conditions allowed the amplification of the modal displacement to took place primarily along the $\mathrm{x}$-axis direction, which was reflected in the optimized solution for both repair patches. The elliptical shape presented a smaller patch than the rectangular one. The total area for the elliptical and rectangular optimized patches was equal to $6,105.7 \mathrm{~mm}^{2}$ and $8,200.2 \mathrm{~mm}^{2}$ respectively.

The total number of iterations until convergence varied considerably for the different cases analyzed. The same goes for the total computational time. This fact is due to the arbitrary definition of the initial estimate for the design variables. Overall, the fastest simulation was the case presented

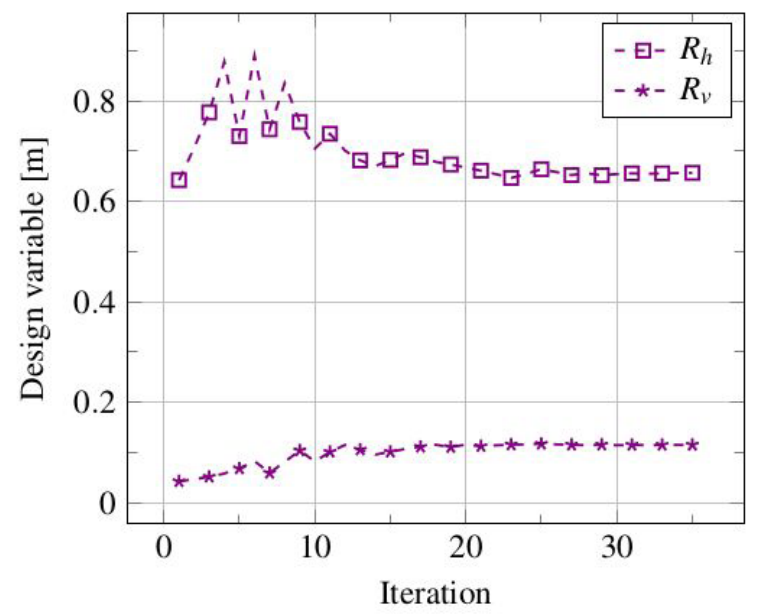

(b) Convergence and optimization evolution.

Figure 7. Elliptical repair patch - SS with an unsymmetrical stacking sequence.

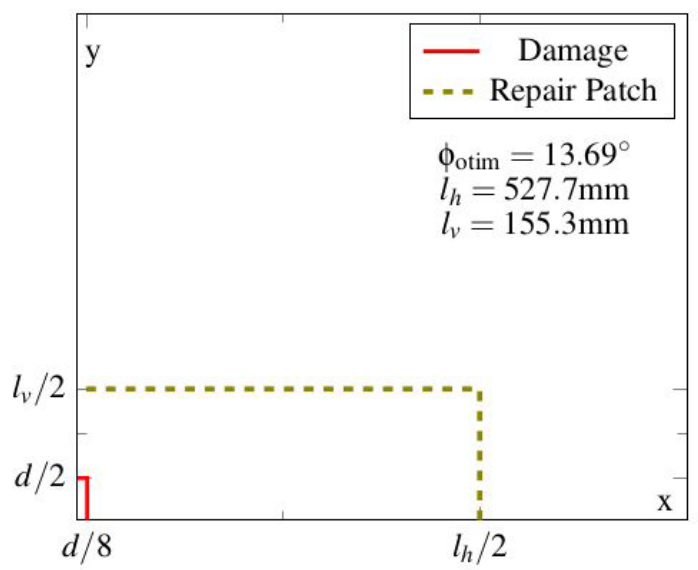

(a) Repair patch and damage region - 1/4 symmetry.

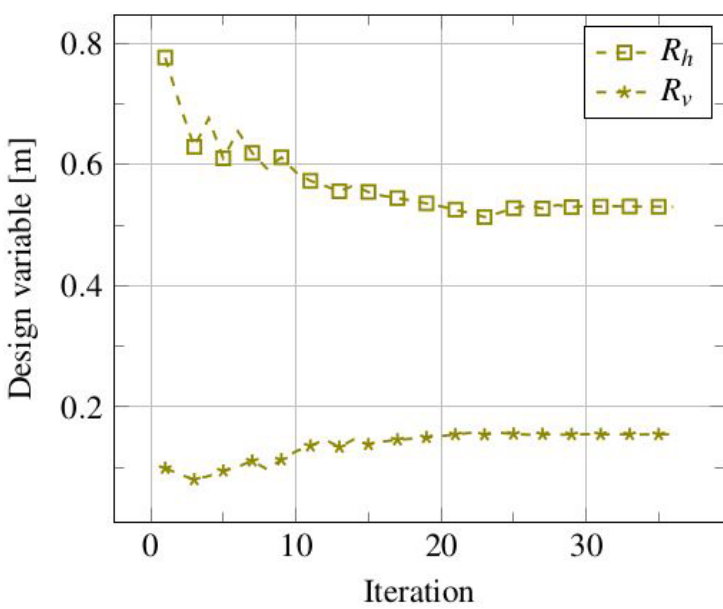

(b) Convergence and optimization evolution.

Figure 8. Rectangular repair patch - SS with an unsymmetrical stacking sequence. 
in Figure 8, which took a total of $8 \mathrm{~m} 9 \mathrm{~s}$ and 35 iterations. The case of Figure 1 was the most time-consuming, taking $12 \mathrm{~m} 15 \mathrm{~s}$ until convergence in a total of 77 iterations.

\section{Final Remarks}

In the restoring of damaged composites with repair patches, an optimum-based methodology for the definition of geometrical parameters $\phi$ and $\Omega_{r}$ appears to be the best answer. The definition of fiber orientation angle as being perpendicular to the damage neutral axis (damage length) has been confirmed inappropriate to repair composites. Besides, the definition of the repair patch orientation is not trivial even for simple and symmetric stacking sequences. Furthermore, the boundary conditions and, mainly, the stacking sequence have a high influence on the shape of optimized repair patches. Each case seems to present a unique modal displacement amplification pattern, and an efficient universal repair approach seems unlikely.

The rectangular and elliptical patches make use of simple geometries, with only two design variables each. Despite this limitation, the results confirmed their efficiency in covering the substrate superficial area suppressing the modal displacement amplitude induced by damage. The patches' versatility could be increased by adding variables to the optimization formulation, such as the patch disposition, and alignment, over the substrate. However, any new variable increases the optimization problem's complexity, and this path should be taken with caution.

In the continuation of the present research, we intend to put these conventional optimized shapes to test in terms of the ultimate strength of repaired panels under flexure. The panel's failure behavior is of great interest. This is especially true for regions encompassing the surroundings of the damaged zone and at the patch edgings. Furthermore, the initiation and propagation of cracks, in the substrate, could give valuable information for a new methodology to determine $\phi$. Instead of evaluating the patch's direction that maximizes the repaired component's strength, $\phi$ could be defined as the direction that minimizes the chance of cracks initiation/propagation.

\section{Acknowledgements}

The authors would like to acknowledge financial support by Brazilian research agencies CAPES and CNPq (grants 431586/2016-0 and 310649/2017-0). Moreover, no potential conflict of interest was reported by the authors.

\section{References}

1. United States of America. U.S. Government Accountability Office-GAO. GAO-11-849: aviation safety: status of FAA's actions to oversee the safety of composite airplanes. Report. Washington, DC; 2011.
2. Katnam K, Silva L, Young T. Bonded repair of composite aircraft structures: a review of scientific challenges and opportunities. Prog Aerosp Sci. 2013;61:26-42.

3. Baker AA. A summary of work on applications of advanced fibre composites at the aeronautical research laboratories, Australia. Composites. 1978;9(1):11-6.

4. Baker AA. Repair of cracked or defective metallic aircraft components with advanced fibre composites an overview of Australian work. Compos Struct. 1984;2(2):153-81.

5. Baker AA, Jones R. Bonded repair of aircraft structures, engineering applications of fracture mechanics. Dordrecht: Martinus Nijhoff Publishers; 1988

6. Baker AA. Bonded composite repair of fatigue-cracked primary aircraft structure. Compos Struct. 1999;47(1-4):431-43.

7. Bachir Bouiadjra B, Fari Bouanani M, Albedah A, Benyahia F, Es-Saheb M. Comparison between rectangular and trapezoidal bonded composite repairs in aircraft structures: a numerical analysis. Mater Des. 2011;32(6):3161-6.

8. Bouchiba MSE, Serier B. A step towards the optimization of composite bonded repair shape using an estimation distribution approach. J Braz Soc Mech Sci Eng. 2016;39:1755-1771.

9. Kumar AM, Hakeem S. Optimum design of symmetric composite patch repair to centre cracked metallic sheet. Compos Struct. 2000;49(3):285-92. http://dx.doi.org/10.1016/S02638223(00)00005-2.

10. Brighenti R, Carpinteri A, Vantadori S. A genetic algorithm applied to optimisation of patch repairs for cracked plates. Comput Methods Appl Mech Eng. 2006;196(1-3):466-75.

11. Rachid M, Serier B, Bouiadjra B, Belhouari M. Numerical analysis of the patch shape effects on the performances of bonded composite repair in aircraft structures. Compos, Part B Eng. 2012;43(2):391-7.

12. Talebi B, Abedian A, Firooz S. Optimization of composite patch repair for inclined crack on aluminum plate using genetic algorithm. In Proceedings of the 30th International Council of Aeronautical Sciences (ICAS); 2016; Daejeon, South Korea. Bonn: ICAS; 2016.

13. Wang X, Dai Y, Huang F. Composite patch bonded repair simulation and optimization of the lap joints. In Proceedings of the First Symposium on Aviation Maintenance and Management Volume II; 2014; China. Berlin: Springer; 2014. p. 507-515.

14. Nadella KS, Cesnik CES. Local interaction simulation approach for modeling wave propagation in composite structures. CEAS Aeronaut J. 2013;4:35-48.

15. Fekih SM, Albedah A, Benyahia F, Belhouari M, Bouiadjra BB, Miloudi A. Optimisation of the sizes of bonded composite repair in aircraft structures. Mater Des. 2012;41:171-6.

16. The MathWorks Inc. MATLAB ${ }^{2}$. version 8.0.0.783 (R2012b). Natick, MA: MathWorks; 2012.

17. Ansys ${ }^{\circledR}$. Academic research mechanical, release 17.0. Canonsburg, P: Ansys Inc.; 2017.

18. Nocedal J, Wright S. Numerical optimization. New York: Springer; 2006. (Springer Series in Operations Research and Financial Engineering).

19. Mehrotra S. On the implementation of a primal-dual interior point method. SIAM J Optim. 1992;2(4):575-601.

20. Arora J. Introduction to optimum design. Amsterdam: Elsevier Science; 2011 